\section{Orbiting nuclear reactors still a problem for astronomers}

\section{Berkeley}

OpPonents of nuclear reactors in Earth orbit have tried to bring gamma-ray astronomers on board, with a promise that a ban on orbiting reactors could end their noise. Joel Primack, a theoretical physicist at the University of California at Santa Cruz and a member of the Federation of American Scientists (FAS), which opposes the use of nuclear reactors in Earth orbit, recently organized a session on orbiting reactors at a workshop on high-resolution gamma-ray cosmology in Los Angeles.

The gamma-ray astronomers may be reluctant to join forces for the ban, but they have nevertheless begun to discuss difficulties they have been experiencing for years with spurious background radiation apparently caused by orbiting Soviet reactors.

Members of gamma-ray detection projects including the High Energy Astronomical Observatory and the Solar Maximum Mission (Solar Max) have for noise ratio. with a detector. problems with spurious background

years noticed spurious bursts of background radiation that complicate their measurements, which under the best of circumstances involve a very low signal to

German scientist Eric Rieger, a collaborator on the Solar Max, was the first to identify the radiation bursts as a discrete component of the background noise. The bursts, which seem to originate from orbiting nuclear reactors on Soviet RORSAT reconnaissance satellites, come in two varieties: gamma rays detected directly from the unshielded reactors, and clusters of positrons that remain trapped in the Earth's magnetic field, and annihilate with a burst of radiation on contact

The US government has apparently known of the phenomenon for several years, but has kept the information classified, says Primack. Last month, Primack saw an unclassified National Aeronoutics and Space Administration (NASA) memo, dated August 1988, that described the radiation bursts and warned that they

\section{Lasker prizes shared by three researchers}

\section{Washington}

THE Albert Lasker Medical Research Awards for 1988 go to a clinical researcher for his work on drug addiction and two molecular biologists for their studies of the role on RNA in living cells.

The medical research award is shared by Thomas Cech of the University of Colorado and Phillip Sharp of the Massachusetts Institute of Technology. Cech's work has shown that polyribonucleotides can act as enzymes, a discovery that Frank Westheimer wrote "is revolutionary in its direct impact on the theory of catalysis, in its promise for future discovery, and its implications with respect to prebiotic chemistry" (Nature 319, 534; 1986). In his work, Sharp showed how genes can be split up by non-coding sequences of DNA,
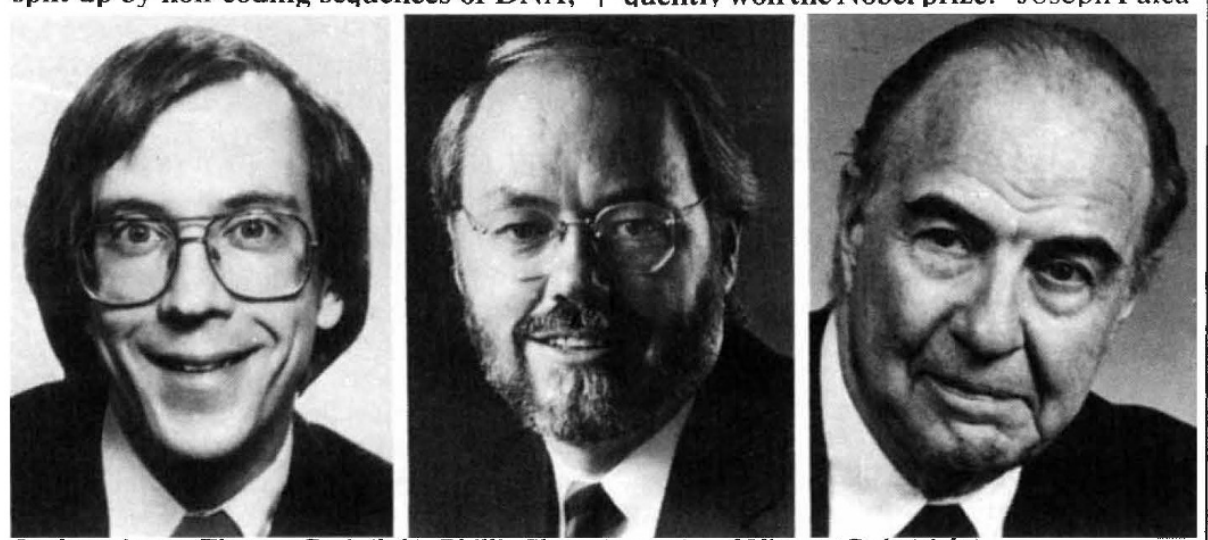

Lasker winners Thomas Cech (left), Phillip Sharp (centre) and Vincent Dole (right). could jeopardize data collection by NASA's planned Gamma Ray Observatory. Primack, who was an invited speaker at the Los Angeles workshop earlier this month, requested time for a second presentation on the proposal for a reactor ban, supported by the FAS and the Committee of Soviet Scientists Against the Nuclear Threat (see Nature 334, 465; 1988).

After Primack's presentation, Solar Max scientist Edward Chupp, of the University of New Hampshire, spoke about problems the bursts had caused for the Solar Maximum Mission, and James Kurfess of the Naval Research Laboratory confirmed that the bursts had been recognized for some time, and their potential influence on the Gamma Ray Observatory were being studied.

Primack says many of the scientists in the audience acknowledged that they too had been troubled by the background, but had never understood its source.

Solar Max project scientist David Forrest, of the University of New Hampshire, says the positron annihilations generated much excitement when they were first identified by Rieger, shortly after the Solar Maximum satellite was launched. But he says it soon became clear from the periodicity of the events that the positrons were from a man-made rather than astronomical source.

Computer programs were developed to identify and cancel the spurious signals, but studying weak gamma-ray sources such as Supernova 1987 a was nevertheless made much more difficult. In addition, Forrest says the events have become dramatically more frequent in the past year, for unknown reasons, occurring as often as once per orbit.

The NASA memo that originally alerted Primack to the problem warns that the bursts could jeopardize the Gamma Ray Observatory by setting off a trigger meant to initiate observation of astronomical sources of gamma-rays. Once activated, the sensor will be unable to respond to gamma-ray bursts from astrophysical events for up to one orbit. The memo asks Gamma Ray Observatory scientists to study the problem and seek means to avert it.

While banning nuclear satellites would be one solution to the problem - and Primack is quick to point out that the 'fleet' of powerful nuclear reactors planned as part of the Strategic Defense Initiative may be devastating to future, more sensitive gamma-ray sensors - the astronomers seem to believe that ways can be found to get around the problem. "Gamma-ray astronomers don't tend to have much influence in controlling the outside environment", says Forrest. The most they can hope for, he says, is to develop a means to recognize and subtract the spurious signals.

Marcia Barinaga 